\title{
GATA4 induces liver fibrosis regression by deactivating hepatic stellate cells
}

\author{
Noelia Arroyo,, Laura Villamayor, ${ }^{1}$ Irene Díaz, ${ }^{1,2}$ Rita Carmona, ${ }^{3,4}$ Mireia Ramos-Rodríguez, ${ }^{5}$ \\ Ramón Muñoz-Chápuli, ${ }^{3}$ Lorenzo Pasquali, ${ }^{5}$ Miguel C. Toscano, ${ }^{6}$ Franz Martín, ${ }^{1,2}$ David A. Cano, ${ }^{7}$ \\ and Anabel Rojas ${ }^{1,2}$ \\ 'Centro Andaluz de Biología Molecular y Medicina Regenerativa (CABIMER), Universidad Pablo de Olavide, Universidad \\ de Sevilla, Consejo Superior de Investigaciones Científicas (CSIC), Seville, Spain. ${ }^{2}$ Centro de Investigación Biomédica en

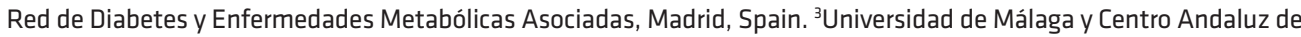 \\ Nanomedicina, Málaga, Spain. ${ }^{4}$ Department of Human Anatomy and Embryology, Legal Medicine and History of Medicine, \\ Faculty of Medicine, University of Málaga, Málaga, Spain. ${ }^{5}$ Endocrine Regulatory Genomics, Department of Experimental \\ \& Health Sciences, University Pompeu Fabra, Barcelona, Spain. ${ }^{6}$ Amarna Therapeutics, Sevilla, Spain. ${ }^{7}$ Instituto de \\ Biomedicina de Sevilla, Hospital Universitario Virgen del Rocío/CSIC/Universidad de Sevilla, Seville, Spain.
}

In response to liver injury, hepatic stellate cells activate and acquire proliferative and contractile features. The regression of liver fibrosis appears to involve the clearance of activated hepatic stellate cells, either by apoptosis or by reversion toward a quiescent-like state, a process called deactivation. Thus, deactivation of active hepatic stellate cells has emerged as a novel and promising therapeutic approach for liver fibrosis. However, our knowledge of the master regulators involved in the deactivation and/or activation of fibrotic hepatic stellate cells is still limited. The transcription factor GATA4 has been previously shown to play an important role in embryonic hepatic stellate cell quiescence. In this work, we show that lack of CATA4 in adult mice caused hepatic stellate cell activation and, consequently, liver fibrosis. During regression of liver fibrosis, Gata4 was reexpressed in deactivated hepatic stellate cells. Overexpression of Gata4 in hepatic stellate cells promoted liver fibrosis regression in $\mathrm{CCl}_{4}$-treated mice. CATA4 induced changes in the expression of fibrogenic and antifibrogenic genes, promoting hepatic stellate cell deactivation. Finally, we show that GATA4 directly repressed EPAS1 transcription in hepatic stellate cells and that stabilization of the HIF2 $\alpha$ protein in hepatic stellate cells leads to liver fibrosis.

Authorship note: NA and LV contributed equally to this study.

Conflict of interest: The authors have declared that no conflict of interest exists.

Copyright: (c) 2021, Arroyo et al. This is an open access article published under the terms of the Creative Commons Attribution 4.0 International License.

Submitted: March 29, 2021 Accepted: October 20, 2021 Published: December 8, 2021

Reference information: /CI Insight. 2021;6(23):e150059. https://doi.org/10.1172/jci. insight.150059.

\section{Introduction}

Liver fibrosis is characterized by the excessive accumulation of extracellular matrix (ECM) components that might eventually lead to cirrhosis and impaired hepatic function. Liver fibrosis is caused by different chronic liver pathologies, including hepatitis $\mathrm{B}$ and $\mathrm{C}$ virus infections, alcohol abuse, and nonalcoholic steatohepatitis (1). Regardless of the etiology, hepatic stellate cells (HSCs) are key players in the development of liver fibrosis. In response to liver injury, HSCs get activated, acquiring proliferative and contractile features and become myofibroblast-like cells that express $\alpha$-smooth muscle actin ( $\alpha$-SMA) and synthesize ECM components such as collagen and laminin (2). The initial activation of HSCs is caused by paracrine signals from other liver cells, such as macrophages, hepatocytes, and endothelial cells (3). However, the maintenance of the active state of HSCs requires autocrine as well as paracrine loops (4). Among the signaling molecules shown to induce activation of HSCs are PDGF- $\beta$, TGF- $\beta$, and inflammatory cytokines (5). It has become increasingly clear in recent years that liver fibrosis can be reversed upon cessation of injury. The regression of liver fibrosis seems to involve the clearance of activated HSCs (aHSCs; refs. 6, 7). Lineage-tracing studies in mouse models have demonstrated that, during the regression of liver fibrosis, approximately half of the aHSCs escape from apoptosis or cell senescence and revert their phenotype to an inactive or quiescent-like state, a process called deactivation (8). This deactivation phenomenon has also been recently described in human HSCs (9). In this context, targeting the deactivation of aHSCs has emerged as a novel and promising therapeutic approach for liver fibrosis.

However, our knowledge of the underlying molecular mechanisms, in particular master regulators, involved in aHSC deactivation is still limited. We have previously shown the zinc finger type transcription factor GATA4 plays a critical role in HSC phenotype during embryonic development. Inactivation of 
Gata4 specifically in HSCs results in aberrant accumulation of ECM components and activation of HSCs in embryonic livers (10). Here, we aimed to determine the role of GATA4 in HSC deactivation during adult liver fibrosis regression. We show that GATA4 induced the deactivation of adult aHSCs. We also demonstrate that GATA4 regulated the expression of the endothelial PAS domain-containing protein 1 gene (EPAS1) and that EPAS1/HIF2 $\alpha$ activity in HSCs induces liver fibrosis in mice.

\section{Results}

GATA4 prevents adult HSC activation. Our previous work demonstrated that Gata4 inactivation in embryonic HSCs, using the G2-Cre line, leads to liver fibrosis, and therefore, this transcription factor is required to maintain the quiescence of embryonic HSCs (10). The G2-Cre line directs the Cre recombinase in the septum transversum and its derivatives, including HSCs and epicardial cells. The embryonic lethality of G2-Cre; Gata $4^{\text {Ilfl }}$ mice precludes the study of GATA4 function in adult HSCs. To determine whether GATA4 regulates adult HSC quiescence in mice, we used an adenovirus-mediated system to deliver the Cre recombinase. Gata $^{\text {Ilfl }}$ adult mice were injected with Cre-expressing adenoviruses (Ad-Cre) via the tail vein. GFP-expressing adenoviruses (Ad-GFP) were used as control. Mice were sacrificed for analysis 2 weeks after adenovirus injections. Previous studies have shown that adenovirus injection in the tail vein infects mostly and robustly liver cells, including hepatocytes, Kupffer cells, and HSCs (11). Indeed, quantification of GFP/desmin-positive cells in the livers of mice injected with $4.6 \times 10^{8}$ viral genome of Ad-GFP revealed an efficient targeting of adenoviruses in HSCs. Around 51\% of HSCs were transduced with Ad-GFP, whereas 14\% were observed in hepatocytes and $11 \%$ in endothelial cells (Supplemental Figure 1; supplemental material available online with this article; https://doi.org/10.1172/jci.insight.150059DS1).

Gata4-floxed mice treated with Ad-Cre displayed a marked decrease in Gata4 mRNA levels in liver, indicating an efficient inactivation of the Gata4-floxed allele by the Cre recombinase (Figure 1A). Efficient excision of the Gata4-floxed allele was further confirmed by PCR of genomic liver DNA (Supplemental Figure 1). Of note, GATA4 accumulation at the protein level could not be properly analyzed in Gata4-floxed mice. Additionally, the identification of the truncated version of the GATA4 protein (which is encoded by this particular floxed allele; ref. 12) with commercial antibodies was not reliable. Inactivation of Gata4 led to an increase in collagen (stained with Sirius red) and laminin accumulation (Figure 1, B, D, E, and G) in the livers of Ad-Cre-treated mice compared with Gata4-floxed mice injected with Ad-GFP (Figure 1, B, C, E, and F). The accumulation of ECM in Gata4-floxed mice injected with Ad-Cre was concomitant with HSC activation, as demonstrated by the prominent expression of $\alpha$-Sma (Figure 1, H and J), compared with mice treated with Ad-GFP (Figure 1, $\mathrm{H}$ and I). Quantitative RT-PCR analysis confirmed the increase in the expression of fibrotic markers, including collagen 1A1 (Col1A1), Acta2, Timp1, and Tgf $\beta R 1$ (Figure 1, K and L). Conversely, the expression levels of STAT1, a negative regulator of liver fibrosis, were reduced (Figure 1L). Of note, injection of Ad-GFP and Ad-Cre in C56BL/ 6 wild-type mice did not cause any defects in liver histology (Supplemental Figure 1). Altogether, these data indicate that GATA4 is required to maintain the quiescent state of adult HSCs.

Gata4 is reexpressed in HSCs during regression of liver fibrosis. Next, we decided to analyze the Gata4 expression pattern in adult HSCs during the development of liver fibrosis and posterior regression in the $\mathrm{CCl}_{4}$-induced liver injury model. To determine whether Gata4 is reexpressed in HSCs during the regression of liver fibrosis, we performed lineage-tracing analysis by crossing the HSC-specific G2-Cre mouse line with ROSA26R-eYFP mice. At adult stages, the majority of HSCs, as determined by desmin accumulation, expressed YFP (76\%; Supplemental Figure 2). A similar percentage of YFP-labeled cells were positive for GATA4 (Figure 2, A-C and P). As expected, mice treated with $\mathrm{CCl}_{4}$ show increased accumulation of collagen fibers in the liver compared with mice treated with vehicle (Figure 2, D, I, and Q). Immunostaining for collagen IV confirmed accumulation of this ECM protein in CCl4-treated mice compared with oil-treated mice (Figure 2, E and J). Several reports have found that female rodents are more resistant to $\mathrm{CCl}_{4}$ treatment than male rodents $(13,14)$. However, we did not find different susceptibility toward $\mathrm{CCl}_{4}$ between male and female mice, as assessed by liver collagen fiber accumulation or serum ALT and AST levels (Supplemental Figure 3), likely due to the low levels of $\mathrm{CCl}_{4}$ used in this experiment. In agreement with the elevated accumulation of collagen fibers observed in $\mathrm{CCl}_{4}$-treated mice, the expression of CollA1 and the activation marker for HSCs, $\alpha$-Sma, was increased (Figure 2, I, T, and U). A dramatic downregulation of Gata4 expression in $\mathrm{HSC}$ from $\mathrm{CCl}_{4}$-treated mice was observed (Figure 2, F-H, P, S, and V; see complete unedited blots in the supplemental material). After the recovery period ( 1 month after $\mathrm{CCl}_{4}$ treatment), collagen fibers in $\mathrm{CCl}_{4}$-treated mouse livers, including collagen IV, were clearly diminished, 


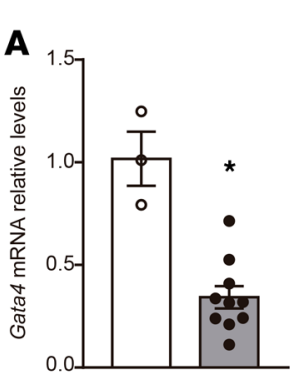

B

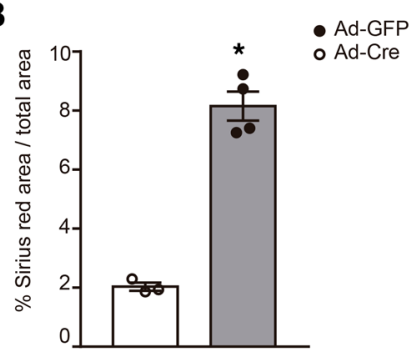

E

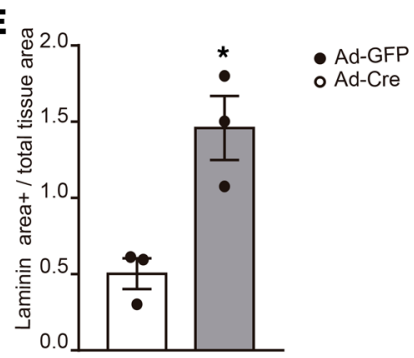

H

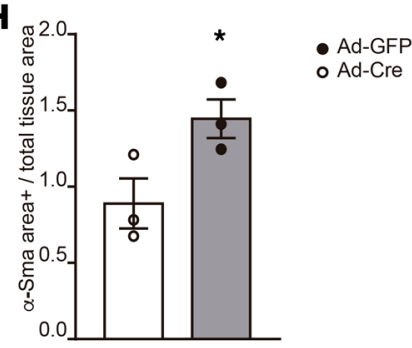

$\mathbf{K}$

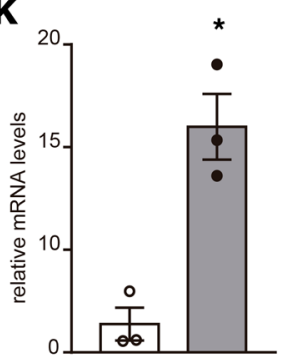

Col1A1
$\mathbf{L}$

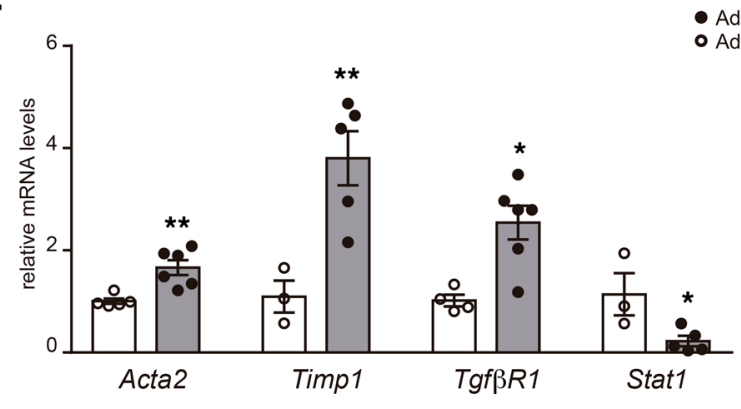

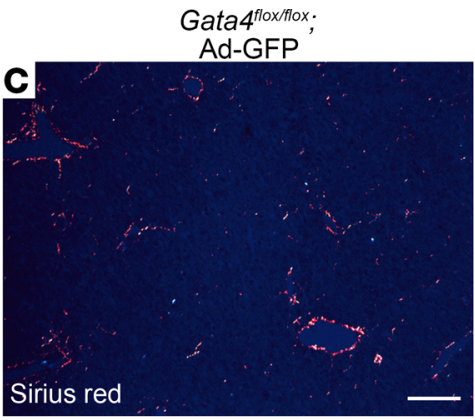
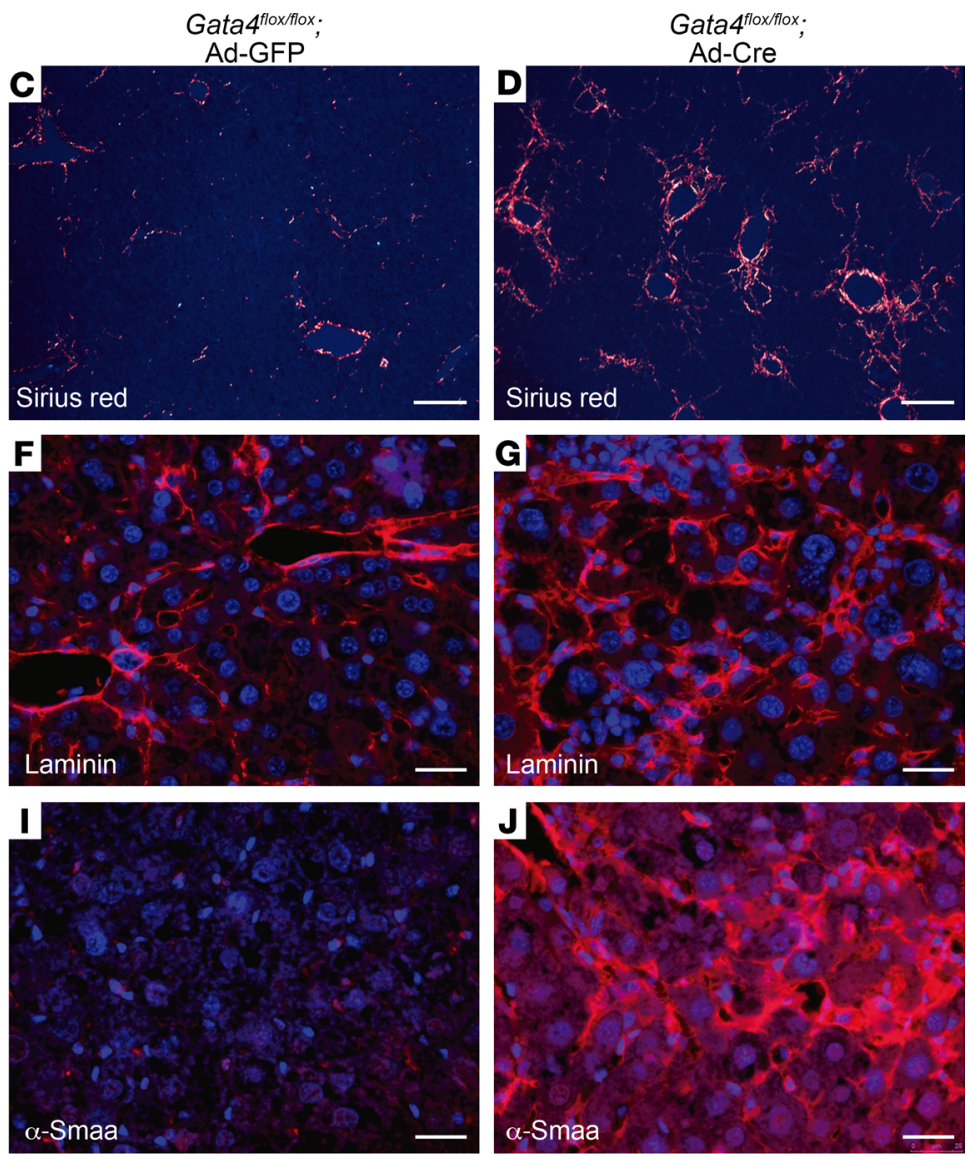

- Ad-GFP Ad-Cre

Figure 1. Gata4 inactivation induces liver fibrosis in adult stages. (A) Quantitative RT-PCR analysis of Gata4 expression in livers of adult Gata4-floxed mice injected with GFP-expressing (Ad-GFP) or Cre-expressing adenoviruses (Ad-Cre) (Ad-GFP $n=3$; Ad-Cre $=10$ ). (B) Quantification of Sirius red-stained area per total liver area in Ad-GFP- and Ad-Cre-injected mice ( $n=3$, Ad-GFP; $n=4$, Ad-Cre). Polarized light microscopy images of Sirius red-stained liver sections from Gata4-floxed mice treated with (C) Ad-GFP and (D) Ad-Cre adenoviruses. (E) Quantification of the liver area immunostained for the ECM protein laminin in Ad-GFP- and Ad-Cre-injected mice ( $n=3$ each group). Increased accumulation of the ECM protein laminin in the liver of (F) Ad-GFP- and (G) Ad-Cre-injected mice ( $n=3$, Ad-GFP; $n=4$, Ad-Cre). (H) Quantification of the liver area immunostained for $\alpha$-smooth muscle actin (SMA) in Ad-GFP- and Ad-Cre-injected mice ( $n=3$ each group). Activation of HSCs, marked by $\alpha$-smooth muscle actin is observed in (J) Gata4-floxed mice treated with Ad-Cre adenoviruses compared with (I) Gata4-floxed mice treated with Ad-GFP adenoviruses. Quantitative RT-PCR analysis of (K) Col1A1 and (L) Acta2, Timp1, TgfBR1, and Stat1 expression in livers of Ad-GFP- and Ad-Cre-injected mice ( $n=3-5$, Ad-GFP; $n=5-6$, Ad-Cre). Scale bars: $100 \mu \mathrm{m}$ (C and D); $25 \mu \mathrm{m}$ (F, G, I, and J). Statistical analyses was performed using 2-tailed Student's test. Error bars represent mean \pm SEM. ${ }^{*} P<0.05,{ }^{*} P<0.01$.

and downregulation of Col1A1 and $\alpha$-Sma expression and protein levels was observed (Figure 2, N, O, Q, $\mathrm{R}$, and $\mathrm{T}-\mathrm{V})$. Remarkably, the reduced expression of Gata4 was fully restored in YFP-labeled deactivated HSCs after the recovery period (Figure 2, K-M, P, S, and V). Analysis of Gata4 expression levels at different time points after the cessation of $\mathrm{CCl}_{4}$ treatment $(2,3$, and 4 weeks) revealed a full reactivation of endogenous GATA4 expression in 2 weeks (Supplemental Figure 4). However, clearance of collagen accumulation was only observed after 4 weeks of recovery (Supplemental Figure 4). These results indicate that Gata4 is reexpressed in aHSCs that return to a quiescent state during the regression of liver fibrosis. 

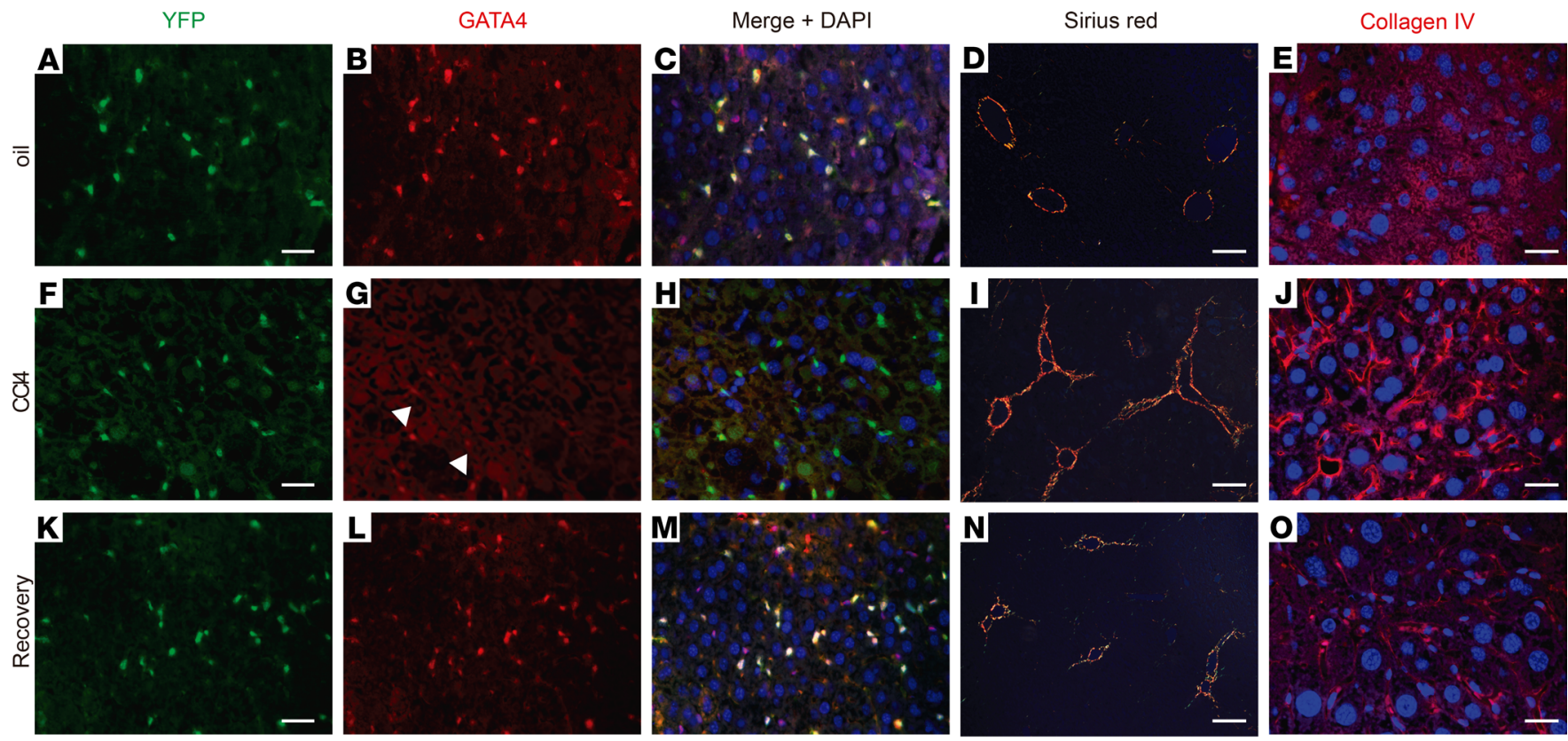

$\mathbf{P}$

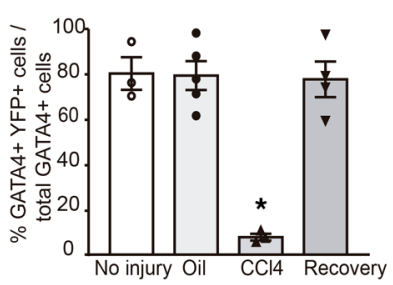

$\mathbf{S}$

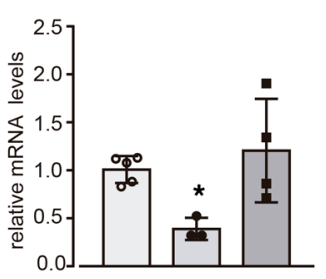

V

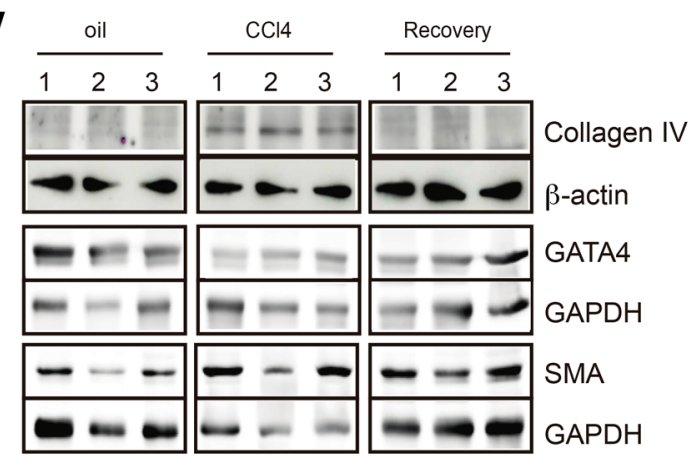

Q
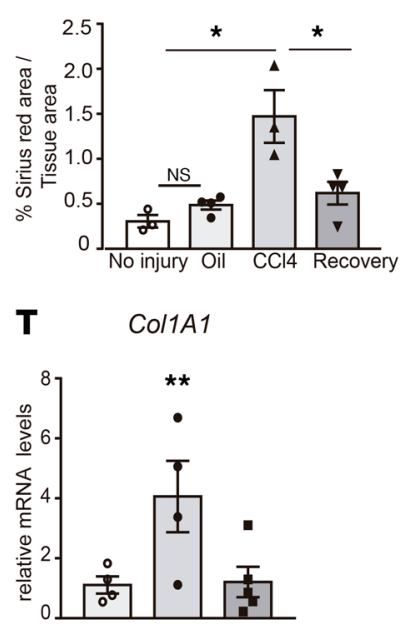

$\mathbf{R}$
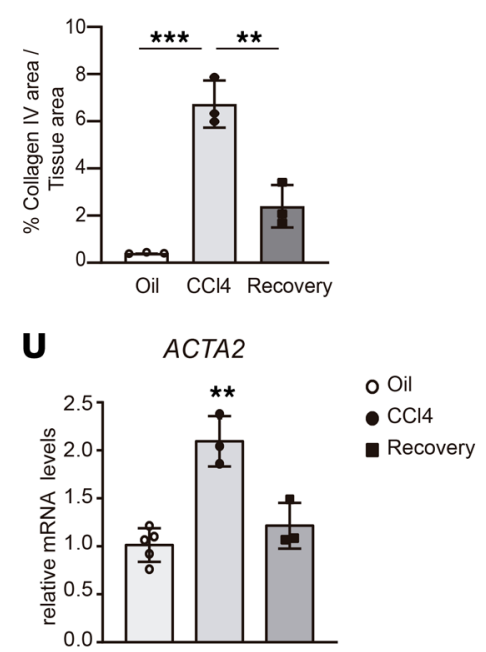

Figure 2. Lineage tracing of G2-Cre-derived cells and Gata4 expression during adult liver fibrosis and recovery. (A-0) Liver sections of G2-Cre; Rosa26ReYFP adult mice. Immunostaining for (A, F, and $\mathbf{K})$ YFP and (B, $\mathbf{G}$, and $\mathbf{L})$ GATA4 in liver sections of mice treated with vehicle (oil) ( $\mathbf{A}$ and $\mathbf{B}$ ) and $\mathrm{CCl}_{4}$ for 4 weeks ( $\mathbf{F}$ and $\mathbf{G}$ ) and 1 month after $\mathrm{CCL}_{4}$ treatment (recovery phase) ( $\mathbf{K}$ and $\mathbf{L}$ ). (C, $\mathbf{H}$, and $\mathbf{M}$ ) Merged images of YFP, GATA4, and DAPI staining. Polarized light microscopy images of Sirius red-stained liver sections of (D) mice treated with oil, (I) mice treated with $\mathrm{CCl}_{4}$, and (N) mice 1 month after recovery. (A-L, $n=3$ each group). Immunostaining for collagen IV in liver sections of (E) mice treated with oil, (J) mice treated with $\mathrm{CCI}_{4}$, and (0) mice 1 month after recovery. (P) Quantification of double GATA4/YFP-positive cells per total GATA-positive cells in livers of each experimental group ( $n=3-5$ each group). (Q) Relative quantification of Sirius red-stained area per total liver area in each experimental group ( $n=3-4$ each group). (R) Relative quantification of collagen IV-stained area per total liver area in each experimental group ( $n=3$ each group). The no injury group denotes mice not injected with adenovirus or CCL4. Quantitative RT-PCR analysis of (S) Gata4, (T) Col1A1, and (U) $\alpha$-Sma expression in each experimental group ( $n=3-5$ each group). (V) Western blot analysis of GATA4, collagen IV, and $\alpha$-SMA accumulation in livers of mice treated with oil, mice treated with $\mathrm{CCI}_{4}$, and mice 1 month after recovery. $\beta$-Actin protein or GAPDH was used for loading control. Samples from 3 independent mice in each experimental group are shown. Scale bars: 25 $\mu \mathrm{m}(\mathbf{A}-\mathbf{C}, \mathbf{F}-\mathbf{H}, \mathbf{K}-\mathbf{M}, \mathbf{E}, \mathbf{J}$, and $\mathbf{0}) ; 100 \mu \mathrm{m}$ (D, I, and $\mathbf{N})$. Statistical analyses was performed using 1-way ANOVA. Error bars represent mean \pm SEM. ${ }^{*} P<0.05$. ${ }^{* *} P<0.01$. 
Gata4 overexpression reduces liver fibrosis in vivo. The results described above suggest a role of GATA4 in the deactivation of adult HSCs during liver fibrosis regression. To test this hypothesis, we evaluated the effect of Gata4 overexpression on liver fibrosis regression in vivo. To this end, mice were treated with $\mathrm{CCl}_{4}$ for 4 weeks to induce liver fibrosis, followed by tail vein injection of Gata4-overexpressing adenovirus (Ad-GATA4) or Ad-GFP as a control. Liver tissues were collected 1 week after adenovirus infection, i.e., 9 days after the last $\mathrm{CCl}_{4}$ injection (Figure 3A). Histological evaluation using Sirius red revealed diminished fibrillar collagen deposition in the livers of mice injected with Ad-GATA4 compared with both control mice (no infection with adenoviruses) and mice injected with Ad-GFP (Figure 3, C, I, L, O, and Q). Decreased CD45 immunostaining indicated reduced liver inflammation in mice injected with Ad-GATA4 (Figure 3, B, H, K, N, and R). Of note, injection of Ad-GATA4 in wild-type mice did not have any effect on collagen accumulation or liver inflammation (Figure 3, E-G, Q, and R). We evaluated $\mathrm{HSC}$ activation in $\mathrm{CCl}_{4}$-treated mice by $\alpha$-SMA immunohistochemistry. Livers of $\mathrm{CCl}_{4}$-treated mice injected with Ad-GATA4 showed a decreased number of $\alpha$-SMA-positive areas compared with both control mice (no infection with adenoviruses) and mice injected with Ad-GFP (Figure 3, D, J, M, P, and S). Quantification of Col1A1 and $\alpha$-Sma mRNA levels confirmed a reduction of fibrosis and HSC activation in Gata4-overexpressing mice (Figure 3, T-V). Thus, Gata4 overexpression promotes liver fibrosis regression in vivo by deactivating HSCs.

GATA4 reverts the active phenotype of HSCs by modulating the expression of fibrogenic and antifibrogenic genes. To confirm the cell-autonomous role of GATA4 in HSC deactivation and to get insight into the underlying molecular mechanisms, we turned to cell culture using LX2 cells, a human HSC cell line that recapitulates many features of the aHSC phenotype. We devised a system that allows robust Gata4 activation in LX2 cells using Ad-GATA4 or Ad-GFP as a control. Adenovirus-mediated overexpression of Gata4 caused a marked reduction in laminin immunoreactivity, which further decreased as a multiplicity of infection (MOI) increased (Figure 4, B and C). At a MOI of 100, nearly $98 \%$ of LX2 cells were transduced. Transduction of LX2 with Ad-GFP at a MOI of 100 did not affect laminin expression (Figure 4A). Next, microarray analyses were performed to analyze changes in gene expression in Gata4-overexpressing LX2 cells compared with GFP-overexpressing LX2 cells (Figure 4D). Among the most downregulated genes were profibrogenic genes such as ECM components (ACTA2, LAMA1, and COLIA1), metalloprotease inhibitors (TIMP1), TGF- $\beta$ receptors (TGF $\beta R 1$ and TGF $\beta R 2)$ and PDGF receptors (PDGFRA and PDGRFB), TLR4, and IL6. Conversely, an increase in the expression of known antifibrogenic factors, such as STAT1, SMAD7, and the transcription factor TCF21, was observed. Quantitative RT-PCR and Western blot analysis confirmed the changes in expression in these fibrosis-related genes (Figure 4, E-G). Interestingly, these genes showed the reverse expression pattern in the liver tissue of embryos lacking Gata4 specifically in HSCs (10), confirming that GATA4 regulates the expression of multiple genes involved in liver fibrosis (Figure 4, $\mathrm{H}$ and I).

EPAS1 is a direct target of GATA4. One of the most downregulated genes in the microarray data was EPAS1, encoding the hypoxia-inducible factor HIF2 $\alpha$. Quantitative RT-PCR analysis confirmed the decreased expression of EPAS1 in Gata4-overexpressing LX2 cells (Figure 5A). Interestingly, Epas1 expression was increased in the liver tissue of embryos lacking Gata4 specifically in HSCs as well as in the livers of adult Gata4-floxed mice treated with Ad-Cre, further supporting an association between GATA4 and Epas1 expression (Figure 5, B and C). To directly evaluate the role of HIF2 $\alpha$ in HSC function, we performed knockdown experiments in LX2 cells using a specific siRNA against EPAS1. LX2 cells transfected with the EPAS1 siRNA exhibited downregulation of the fibrotic markers ACTA2, collagen, and laminin (Figure 5D), revealing a role of HIF2 $\alpha$ in the regulation of HSCs phenotype. Bioinformatic analyses of the EPAS1 conserved regions identified 2 GATA4-binding sites within the first intron, localized at $9717 \mathrm{bp}$ after the transcriptional start site (Figure 5E), suggesting that EPAS1 was a direct target of GATA4. ChIP qPCR analysis validated these sites as GATA bona fide binding sites (Figure 5F). To examine the ability of GATA4 to repress the Epas1 promoter, we generated reporter constructs containing the intronic region of Epas 1 with the wild-type version of the 2 GATA sites or the mutated version (Figure 5E) fused to the luciferase gene to generate the pGL3-Epas1 wt and pGL3-Epas1 mut reporter plasmids, respectively. We have previously reported endogenous expression of Gata4 in 293T cells (15). Transient transfection of the pGL3-Epas1 wt reporter plasmid in 293T cells resulted in significantly lower luciferase activity compared with 293 T cells transfected with pGL3-Epas1mut reporter plasmid (Figure 5G). Altogether, these results indicate that GATA4 directly represses EPAS1 transcription through the 2 identified GATA sites.

Stabilization of HIF2 $\alpha$ induces liver fibrosis. The results described above suggest that EPAS1 expression was associated with HSC activation and liver fibrosis. We decided to evaluate the biological role of HIF $2 \alpha$ in 
A
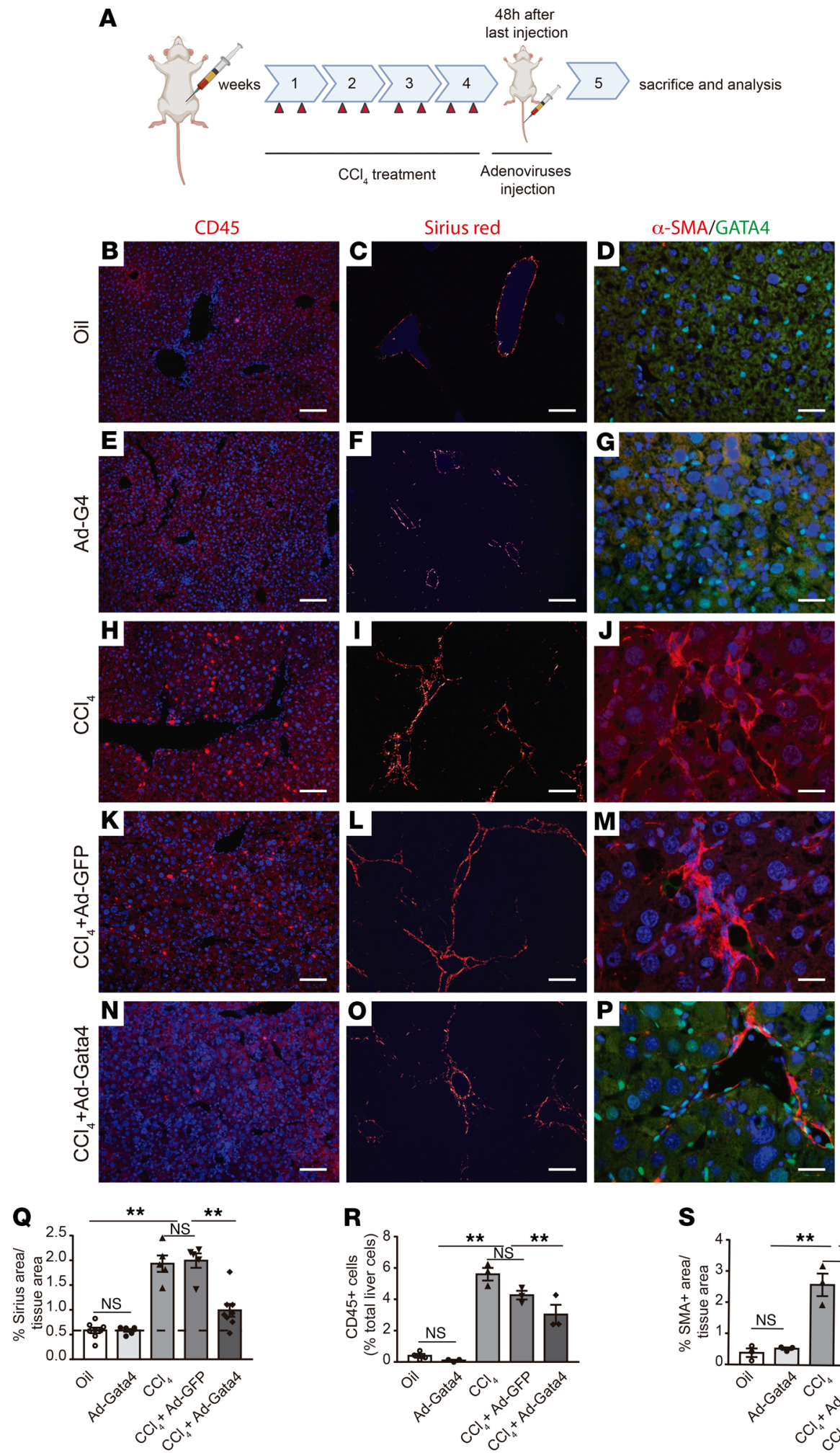

$\mathbf{R}$
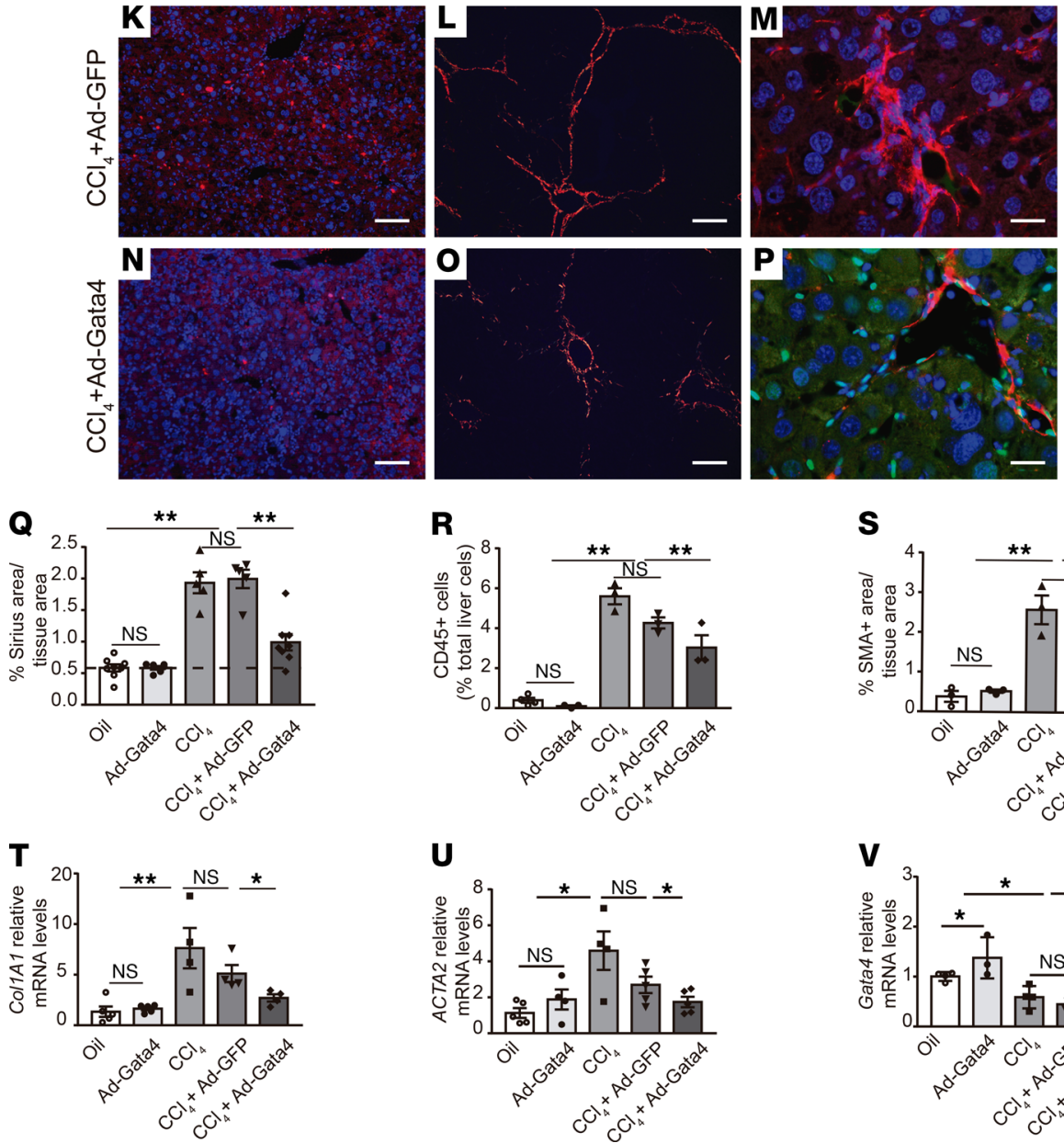

U

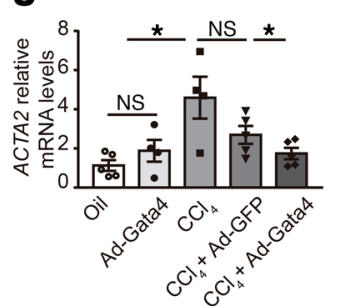

$\mathbf{s}$

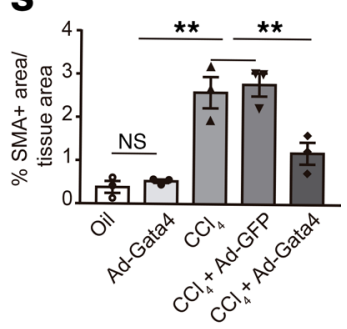

V

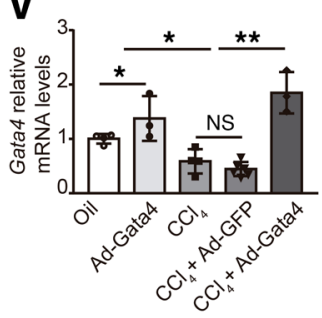

Figure 3. GATA4 promotes the regression of liver fibrosis regression. (A) Schematic of the experimental design. To induce liver fibrosis, $\mathrm{CCl}_{4}$ was administered to adult wild-type C57BL/ 6 mice for 4 weeks. Two days after the last $\mathrm{CCl}_{4}$ injection, mice were infected with GFP-expressing (Ad-GFP) and Gata4-overexpressing (Ad-Gata4) adenoviruses via the tail vein. Liver tissue is analyzed 1 week after adenovirus infection. Wild-type C57BL/6 mice treated with $\mathrm{CCL}_{4}$ showed an increase in inflammatory cells, (H) marked by CD45 immunostaining, (I) accumulation of collagen fibers marked with Sirius red staining, and $(\mathrm{J})$ activation of HSCs, marked by $\alpha$-SMA accumulation, compared with control mice treated with vehicle (oil) (B, C, and D, respectively). Infection of Ad-GATA4 adenoviruses in wild-type untreated C57BL/6 mice did not effect (E) CD45 cell numbers, (F) collagen accumulation, and (G) $\alpha$-SMA accumulation. Increased numbers of CD45-positive cells, $\alpha$-SMA expression in HSCs and collagen accumulation were observed in $\mathrm{CCl}_{4}$-treated mice followed by administration of Ad-GFP adenoviruses (K-M and $\mathbf{Q}-\mathbf{U})$. A remarkable decrease in $(\mathbf{N})$ the number of inflammatory cells, (O) collagen accumulation, and (P) HSC activation was observed after administration of Ad-CATA4 adenoviruses to $\mathrm{CCl}_{4}$-treated mice compared with $\mathrm{CCl}_{4}$-treated mice infected with Ad-GFP adenoviruses (K, $\mathbf{L}$, and $\mathbf{M}$, respectively). (Q) Relative quantification of Sirius red-stained area per total liver area in each experimental group $(n=5-8)$. (R) Quantification of CD45-positive cells per total liver cells in each experimental group ( $n$ = 3 each group). (S) Relative quantification of $\alpha$-SMA-positive area per total liver area in each experimental group ( $n=3$ each group). Quantitative RT-PCR analysis of (T) Col1A1 ( $n=4-5$ each group), (U) $\alpha$-Sma ( $n=4-5$ each group), and (V) Gata4 ( $n=3-5$ each group) expression in each experimental group. Scale bars: $100 \mu \mathrm{m}$ (B, C, E, $\mathbf{F}, \mathbf{H}, \mathbf{I}, \mathbf{K}, \mathbf{L}, \mathbf{N}$, and $\mathbf{0}$ ); $25 \mu \mathrm{m}$ (D, $\mathbf{G}, \mathbf{J}, \mathbf{M}$, and $\mathbf{P})$. Statistical analyses was performed using 1-way ANOVA. Error bars represent mean \pm SEM. ${ }^{*} P<0.05,{ }^{*} P<0.01$. 
Ad-GFP
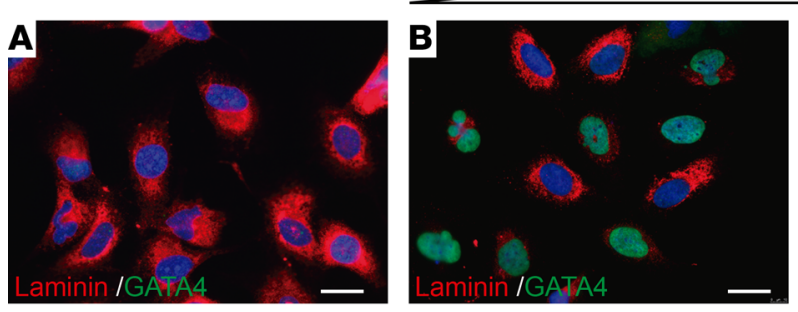

Ad-G

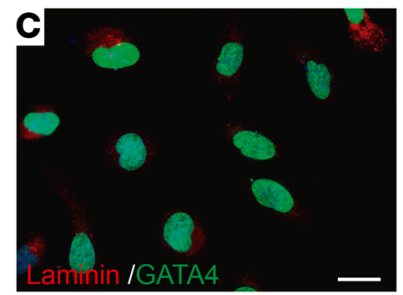

$\mathbf{E}$

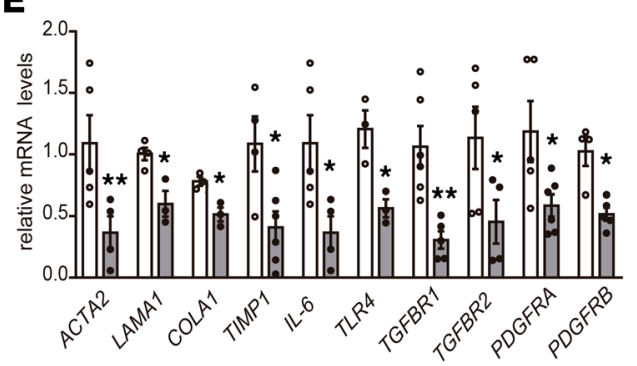

H

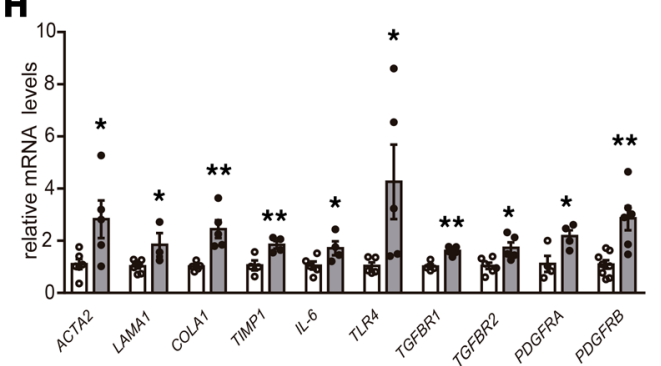

$\mathbf{F}$

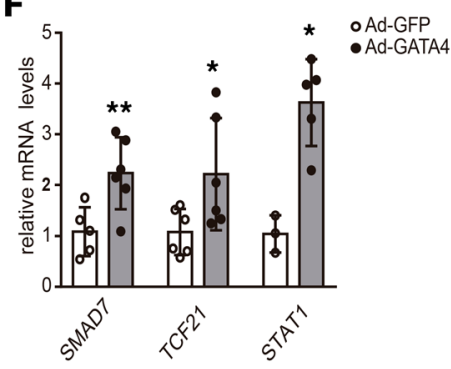

I

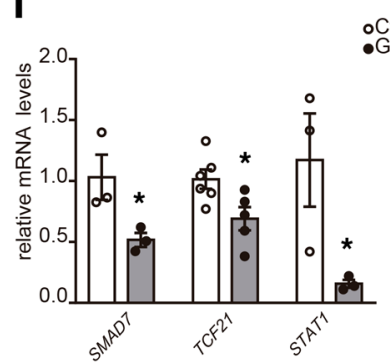

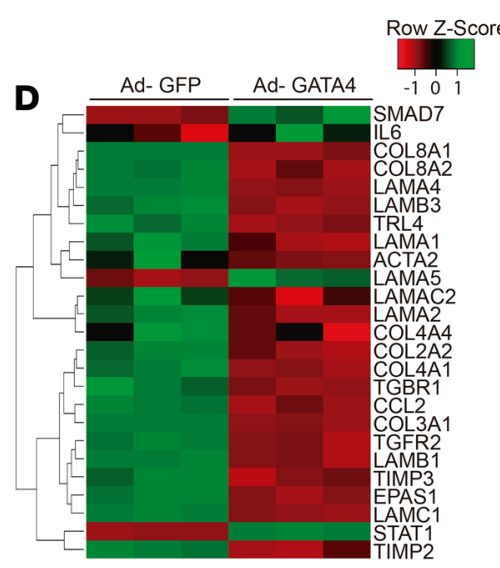

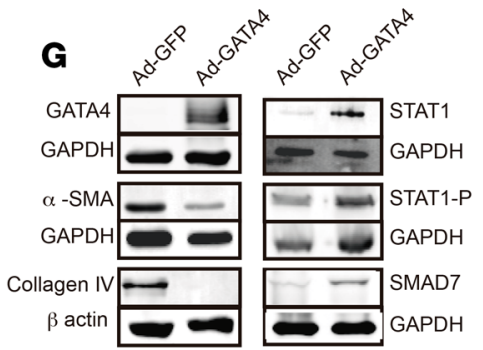

Figure 4. GATA4 regulates the expression of fibrogenic and antifibrogenic genes in HSCs. Immunofluorescence analyses of laminin and GATA4 accumulation in LX2 cells transfected with (A) GFP-expressing (Ad-GFP) (multiplicity of infection [MOI], $=100$ ) and GATA4-expressing (Ad-GATA4) adenovirus at (B) $\mathrm{MOI}=50$ and (C) $\mathrm{MOI}=100$. (D) Heatmap showing the most differentially expressed genes in Ad-GATA4-transfected LX2 cells compared with LX2 cells transfected with Ad-GFP. Validation by quantitative RT-PCR analysis of differentially (E) fibrogenic $(n=3-6)$ and $(\mathbf{F})$ antifibrogenic $(n=3-6)$ expressed genes (Ad-GATA4 vs. Ad-GFP infected LX2 cells) identified in the microarray analyses. (C) Validation by Western blot analysis of selected differentially expressed genes (Ad-GATA4 vs. Ad-GFP infected LX2 cells) identified in the microarray analyses. $\beta$-Actin and GAPDH proteins were used as loading controls. Quantitative RT-PCR analysis of $(\mathbf{H})$ fibrogenic $(n=4-8)$ and $(\mathbf{I})$ antifibrogenic genes $(n=3-6)$ in G2-Cre;Gata4 KO E13.5 embryonic liver. Scale bars: 25 $\mu \mathrm{m}$. Statistical analyses was performed using 2-tailed Student's test. Error bars represent mean $\pm \mathrm{SEM} .{ }^{*} P<0.05,{ }^{*} P<0.01$.

HSC function in vivo. To test this hypothesis in vivo, we specifically activated HIF $2 \alpha$ in HSCs by crossing the HSC-specific G2-Cre mouse line with HIF2dPA mice (G2-Cre;HIF2dPA mice; ref. 16). HIF2dPA mice harbor a modified version of the human HIF2 $\alpha$ that escapes recognition by the von Hippel-Lindau protein (i.e., it is not degraded under normal oxygen levels). Upon Cre recombination, excision of a floxed stop cassette allows the expression of the nondegradable form of HIF2 $\alpha$. G2-Cre;HIF2dPA mice displayed embryonic lethality between E15.5 and E17.5, likely due to cardiac defects (Supplemental Figure 5). The analysis of G2-Cre;HIF2dPA developing hearts at E13.5 revealed thin myocardium, disorganized trabeculae, and regions of the epicardium that stretched away from the underlying myocardium compared with control mice (HIF2dPA mice; Supplemental Figure 5).

A robust accumulation of HIF2 $\alpha$ specifically in HSCs of E13.5 G2-Cre;HIF2dPA livers was observed by immunofluorescence analysis (Figure 6, A and B). Gross morphological examination of G2-Cre;HIF2dPA liver at E13.5 showed reduced size compared with control mice (Figure 6, C and D). The decreased in liver size might be explained by the decrease in cell proliferation as well as increase in cell apoptosis observed in E13.5 and E15.5 G2-Cre;HIF2dPA embryonic livers compared with control livers (Figure 6, O and P, and Supplemental Figure 6). Cell proliferation analysis revealed a decrease in both hepatocytes and HSCs of 
A

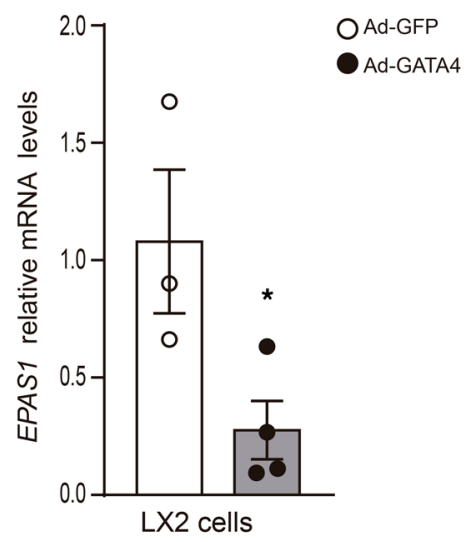

B

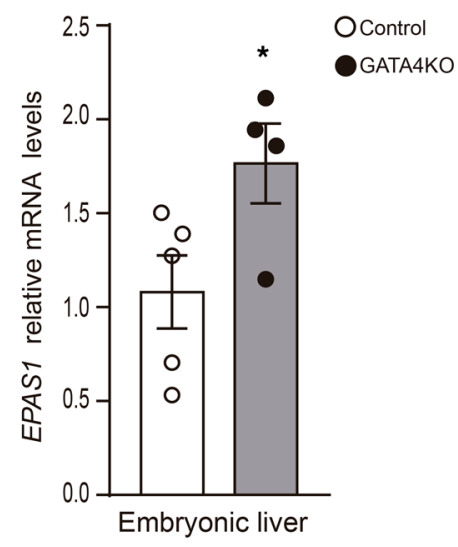

E

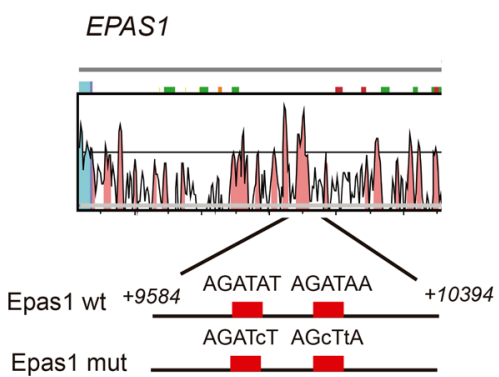

C

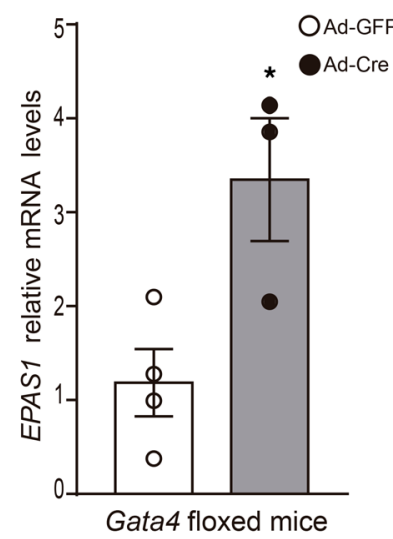

$\mathbf{F}$

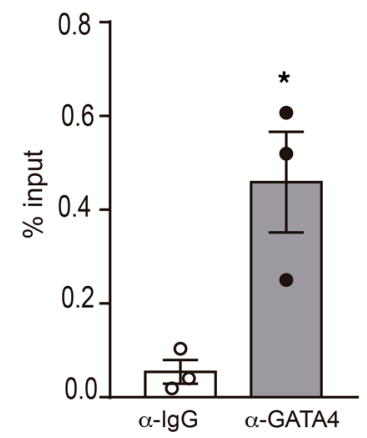

\section{G}

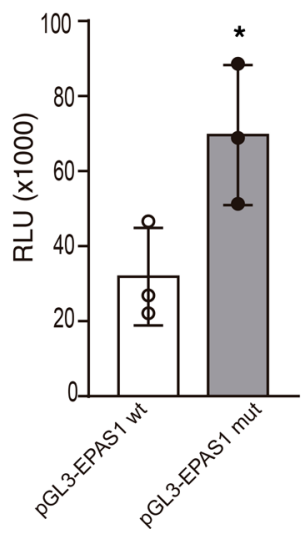

- control siRNA

- EPAS1 siRnA

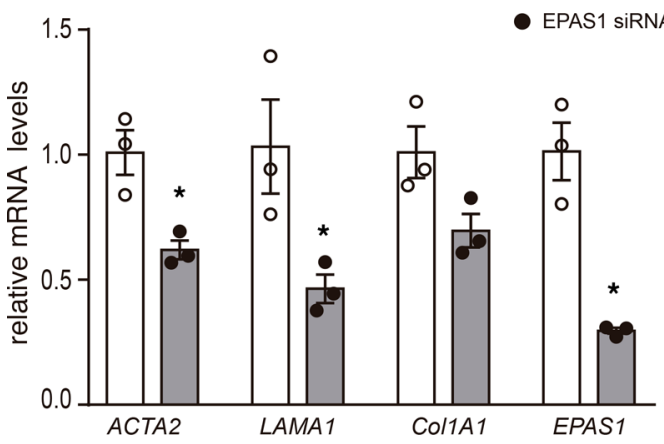

Figure 5. GATA4 is a direct repressor of EPAS1 in HSCs. (A) Quantitative RT-PCR analysis of EPAS1 expression in LX2 cells transfected with GFP-expressing (Ad-GFP) and GATA4-expressing (Ad-GATA4) adenoviruses $(n=$ 3, Ad-GFP; $n=4$, Ad-GATA4). (B) Quantitative RT-PCR analysis of EPAS1 expression in G2-Cre; Gata4 KO E13.5 embryonic liver ( $n=5$, control; $n=4$, GATA4 KO). (C) Quantitative RT-PCR analysis of EPAS1 expression in livers of adult Gata4-floxed mice injected with GFP-expressing (Ad-GFP) $(n=4)$ and Cre-expressing adenoviruses (Ad-Cre) ( $n=3)$. (D) Quantitative RT-PCR analysis in LX2 cells transfected with a siRNA directed against EPAS1 compared with control LX2 cells (treated with siRNA-negative control) $(n=3)$. (E) Schematic of the human EPAS1 intronic region containing 2 conserved GATA sites using Vista Tools software. The blue peak indicates the human EPAS1 transcription start, and the pink peaks indicate conserved noncoding regions between human and mouse. An 810 bp fragment of EPAS1 intronic region containing the 2 conserved GATA4 sites (EPAS1 wt) or the mutated version (EPAS1 mut) was cloned into the pGL3 luciferase vector for reporter assays. The nucleotides mutated in EPAS1 mut are shown in lowercase. The numbers indicate the localization in the EPAS1 locus from the transcriptional start site. (F) ChIP of LX2-overexpressing GATA4 using a GATA4-specific antibody or a IgG-unspecific antibody $(n=3)$. (C) In vitro luciferase reporter assays in 293T cells of $p$ CL3-EPAS1 $w t$ and $p G L 3-E P A S 1$ mut plasmids $(n=3)$. Statistical analyses was performed using 2-tailed Student's test. Error bars represent mean \pm SEM. ${ }^{*} P<0.05$. RLU, relative light units.

embryonic G2-Cre;HIF2dPA livers compared with control livers (Supplemental Figure 6). The histological analyses of E13.5 G2-Cre;HIF2dPA livers revealed disrupted architecture with dispersed hepatocytes (Figure 6, E and F). Indeed, a marked increase in laminin and collagen was seen in G2-Cre;HIF2dPA embryonic livers (Figure 6, G-L and Q). In agreement with the increase in collagen fibers, a dramatic expansion in the number of $\alpha$-SMA-positive cells was found in G2-Cre;HIF2dPA livers (Figure 6, M and N). Quantification of mRNA levels for ECM and $\alpha$-SMA confirmed the activation of HSCs and liver fibrosis (Figure 6R). A remarkably increase in VEGFA expression, a downstream target of HIF2 $\alpha$, was observed in the livers of G2-Cre;HIF2dPA mice (Figure 6R). Altogether, these results indicate that HIF2 $\alpha$ activation causes HSCs to become activated leading to a fibrogenic process. 


\section{Discussion}

Here, we show that GATA4 is a critical regulator of adult HSC quiescence and, therefore, plays a fundamental role in liver fibrosis. We also found that GATA4 directly regulates EPAS1 expression. Furthermore, we uncover a possibly previously unknown function of EPAS1 in HSC activation.

We have previously shown that Gata4 inactivation in embryonic HSCs results in HSC activation. Here, we demonstrate that GATA4 prevents activation of HSCs during adult stages. We have previously generated a Cre line ( $\mathrm{G} 2-\mathrm{Cre}$ ) that targets HSCs very efficiently during embryonic stages (10). However, it also targets epicardial cells and, thus, it might cause extrahepatic defects. To directly test the role of GATA4 in adult HSC activation in vivo we used an adenovirus-mediated strategy. This has been previously proved to be a useful method to genetically manipulate transcription factors in HSCs in vivo (17). In concordance with previous reports, adenovirus infection achieved robust targeting of HSCs (51\%) (17). Using this adenovirus-mediated system we show that inactivation of Gata4 in adult mice caused HSC activation and liver fibrosis. More importantly, we found that adenovirus-mediated Gata4 overexpression in fibrogenic HSCs induced the regression of liver fibrosis by deactivating aHSCs. This is consistent with Gata4 expression in HSCs during liver injury and posterior recovery upon cessation of the insult. During injury, Gata4 expression is lost in aHSCs but, notably, is reexpressed during the HSC deactivation process that takes place through the regression of liver fibrosis. Altogether, our results indicate that reactivation of GATA4 converts aHSC into a quiescent phenotype and, consequently, reverses liver fibrosis.

One potential caveat of the adenovirus approach used in our study is that the observed results might be due to manipulation of GATA4 levels in hepatic cell types other than HSCs. Indeed, hepatocytes are also efficiently targeted by adenoviruses in our experiments. However, even though Gata4 is highly expressed in hepatoblasts during embryonic development its expression in adult hepatocytes is extremely low or almost negligible (18). Moreover, specific inactivation of Gata4 in hepatocytes does not result in any apparent defects in liver morphology and function (18). More recently, it has been reported that specific inactivation of Gata4 in liver sinusoidal endothelial cells (LSECs) using different Cre drivers results in liver fibrosis (19). However, the number of LSECs targeted by adenoviruses in our experiments is very low. Thus, it is unlikely that the liver fibrosis we observed in Gata4-floxed mice treated with Ad-Cre might be due to inactivation of Gata4 in hepatocytes or LSECs rather than HSCs. More importantly, in vitro studies performed with the human HSC LX2 cell line demonstrate that GATA4 can directly revert aHSCs into a quiescent-like, inactivated phenotype.

Our microarray analysis in Gata4-overexpressing LX2 cells revealed a large number of differentially regulated genes related to liver fibrosis. Gata4 overexpression induced downregulation of fibrogenic genes such as ECM components and PDGF and TGF- $\beta$ receptors (5). Interestingly, we observed increased expression of TCF21, a transcription factor that has recently been described to regulate HSC activation (17). Altogether, our data reveal GATA4 as a key component of the transcriptional network regulating adult HSC function. This notion agrees with a very recent report that has identified GATA4 among a group of lineage-specific transcription factors expressed with HSC activation. However, the role of GATA4 in HSC activation was not assessed in vivo in the study (9). Remarkably, the authors found that another member of the GATA family, GATA6, was able to suppress HSC activation in mice. It would be interesting to determine whether GATA4 and GATA6 have complementary roles in HSC biology, as it has shown in other organs.

One of the most interesting genes identified in the gene expression analysis was EPAS1, which encodes the hypoxia-inducible factor HIF $2 \alpha$. HIF $2 \alpha$ is an oxygen-regulated transcription factor that plays a central role in the cellular response to low levels of oxygen (hypoxia). Gata4 overexpression decreased EPAS1 expression in LX2 cells. On the contrary, specific inactivation of GATA4 in HSCs caused an increase in Epas 1 expression in embryonic mouse liver. These results suggested that GATA4 repressed EPAS1 expression. Indeed, in vitro reporter assays confirmed that GATA4 directly repressed EPAS1 expression through 2 conserved GATA sites in an intronic region. Although HIF $\alpha$ subprotein levels are regulated mainly by posttranslational mechanisms, transcriptional regulation of Hif2 $\alpha$ has been reported in different cell types. For example, PGC-1 regulates Hifa transcription in muscle fibers and the PI3K/mTORC2 pathway induces transcriptional activation of Hif2 $\alpha$ in neuroblastoma cells (20). GATA factors can act as activators or repressors in a gene-dependent manner, usually by interacting with other cofactors in specific tissues $(21,22)$. It would be interesting to determine how GATA4 might interact with other cofactors to regulate the specific transcriptional gene network controlling HSC quiescence.

HIF factors have been associated with several hepatic pathologies, including liver fibrosis. Thus, a profibrogenic effect of HIF1 $\alpha$ (another HIF $\alpha$ isoform) has been reported in experimental mouse models of 

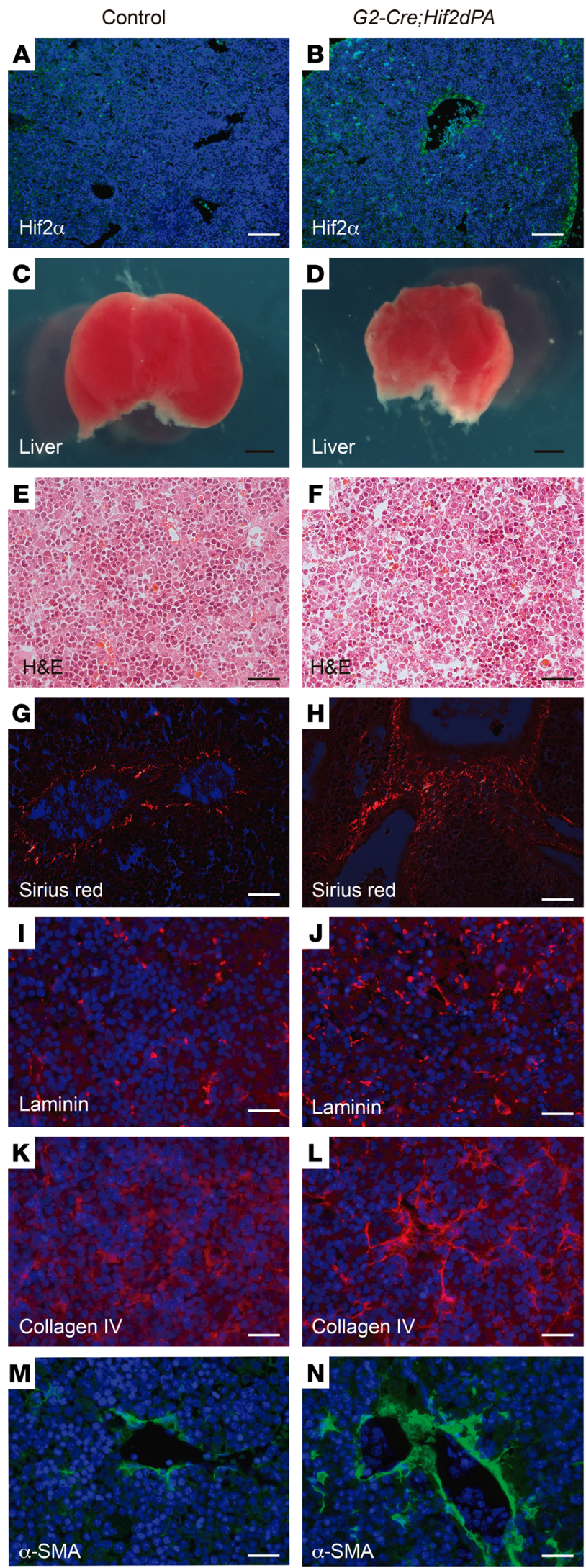
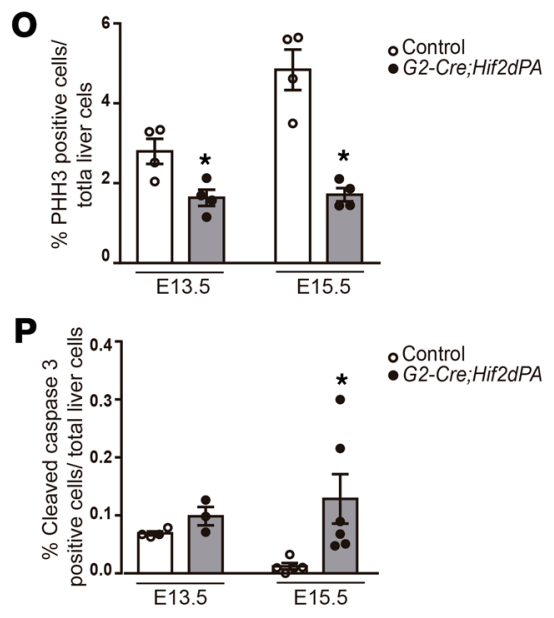

Q

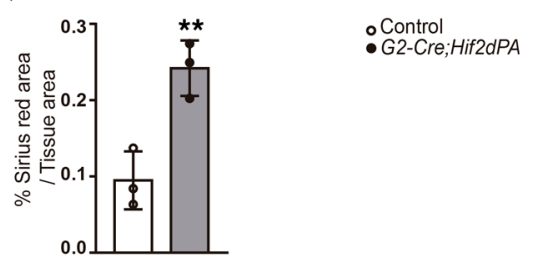

$\mathbf{R}$

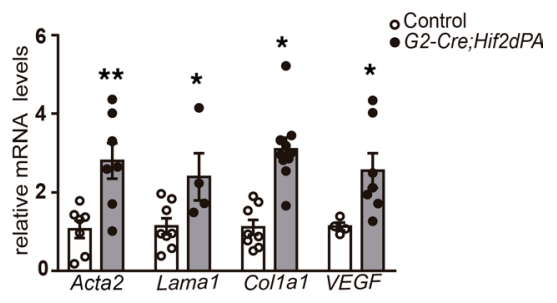

Figure 6. Stabilization of HIF2 $\alpha$ protein in HSCs causes liver fibrosis. Immunofluorescence analysis in liver sections of E13.5 (B) G2-Cre;HIF2dPA embryos demonstrating efficient accumulation of HIF2 $\alpha$ compared with (A) control embryonic livers. Reduced size of E13.5 (D) G2-Cre;HIF2dPA embryonic livers compared with (C) control livers. (E and F) H\&E-stained sections of E13.5 G2-Cre;HIF2dPA and control embryonic livers. ( $\mathbf{G}$ and $\mathbf{H}$ ) Polarized light microscopy images of Sirius redstained liver sections from E13.5 G2-Cre;HIF2dPA and control embryos. Immunofluorescence analysis of (I and J) laminin, ( $\mathbf{K}$ and $\mathbf{L}$ ) collagen IV, and ( $\mathbf{M}$ and $\mathbf{N}$ ) $\alpha$-SMA accumulation in liver sections of E13.5 (J, L, and N) G2-Cre;HIF2dPA and (I, K, and $\mathbf{M}$ ) control embryos. Quantification of proliferating liver cells, (0) marked by phosphohistone H3 immunoreactivity ( $n=4$ each group), and $(\mathbf{P})$ apoptotic liver cells marked by cleaved caspase-3 accumulation (E13.5 $n=3-4$; E15.5 $n=$ 5-6). (Q) Quantification of Sirius red-stained area of liver of E13.5 G2-Cre;HIF2dPA and control embryos ( $n=3$ each group). (R) Quantitative RT-PCR analysis of $\alpha$-Sma, Lama1, Cola1a, and VEGF expression ( $n=5-8)$. Scale bars: $100 \mu \mathrm{m}$ ( $\mathbf{A}$ and B); $500 \mu \mathrm{m}$ (C and D); $25 \mu \mathrm{m}$ (E-N). Statistical analyses was performed using 2-tailed Student's test. Error bars represent mean \pm SEM. ${ }^{*} P<0.05$. 
liver injury. In addition, specific activation of both HIF factors (by genetic inactivation of the $V h l$ gene) in hepatocytes causes inflammation and fibrosis in the liver (23). However, these studies have mostly focused on the role of HIF factors in hepatocytes, but their function in HSCs has been barely investigated. Additionally, it has been reported that hypoxia might occur in the liver during chronic injury and that HIF $2 \alpha$ promotes liver fibrosis and increases the morbidity of nonalcoholic steatohepatitis via upregulation of $\mathrm{NF}-\kappa \beta$ pathway (24). Indeed, it has been established that hypoxia promotes the release of factors from HSCs that may affect the progression of fibrosis (25), but the specific contribution of HIF2 $\alpha$ has not been analyzed. The expression data in LX2 cells and Gata4-deficient mice suggested that increased HIF2 $\alpha$ activity might be associated with HSC activation and liver fibrosis. We confirmed this notion in vivo by activating HIF $2 \alpha$ specifically in embryonic HSCs using the G2-Cre mouse line. We have previously demonstrated that the G2-Cre mouse line efficiently targets HSCs during early embryonic development. Stabilization of HIF $2 \alpha$ induced activation of HSCs and concomitant liver fibrosis as early as E13.5. How HIF2 $\alpha$ activation in HSCs exactly causes liver fibrosis remains to be determined. HIF transcription factors are known to directly regulate a vast number of genes involved in ECM production and vascular remodeling, key biological processes driving organ fibrosis (26). We observed increased expression of VEGF (a well-known HIF2 $\alpha$ target) in G2-Cre;HIF2dPA embryonic liver and VEGF has been reported to promote liver fibrosis (27). Regardless of the mechanisms involved, our results reveal that HIF2 $\alpha$ stabilization might play a role in HSC activation during liver fibrosis. Our results are in concordance with a recent study that has identified EPAS1 as a transcription factor that is upregulated in embryonic HSCs (17). Additional studies in human samples will be necessary to analyze the clinical relevance of HIF $2 \alpha$ accumulation in liver fibrosis, including its potential use as a therapeutic target.

Embryos with HIF2 $\alpha$ activation directed by G2-Cre displayed embryonic lethality. The reasons for this lethality are not entirely clear, but it might be due to cardiac defects. It should be noted that the G2-Cre line also targets the epicardium during cardiac development, and aberrant HIF $\alpha$ activity in the developing heart has been shown to cause cardiac defects and embryonic lethality (28).

In summary, our results indicate that GATA4 is an important master regulator of HSC function. In particular, our data indicate that reactivation of GATA4 in aHSC is crucial to induce the regression of liver fibrosis and, thus, GATA4 may represent a potential therapeutic approach for liver fibrosis.

\section{Methods}

Mice. Gata4 ${ }^{I / f l}$, G2-Cre, ROSA26RYFP, HIF2dPA [Rosa26Sor ${ }^{\mathrm{Tm} 4\left(\mathrm{Hif2}^{*}\right) \text { Kael] }}$, and G2-Cre mice and strategies for genotyping have been previously described (10, 16, 29, 30).

Histology, immunohistochemistry, and immunofluorescence. Dissected livers were fixed in 4\% paraformaldehyde in PBS at $4^{\circ} \mathrm{C}$ overnight and processed for paraffin embedding in a Leica ASP200S tissue processor. Histological analyses and Sirius red staining were performed as described previously (10).

The following primary antibodies were used at the indicated dilutions: rabbit anti-cleaved caspase-3 (1:200, Cell Signaling Technology, 9661); rat anti-CD45 (1:200, BD Pharmingen, BD Biosciences 557390); rabbit anti-collagen IV (1:400, Abcam, Ab19808); goat anti-GFP (1:200, Abcam, Ab6673); mouse anti-GATA4 (1:100, Santa Cruz Biotechnology, SC-25310); goat anti-HNF4 $\alpha$ (1:100, Santa Cruz Biotechnology, SC-6556); rabbit anti-laminin (1:50, MilliporeSigma, L9393); mouse anti-HIF2 $\alpha$ (1:100, Abcam, Ab8365); rabbit anti-Phospho-Histone H3 (1:500, MilliporeSigma, 06-570); mouse anti-Ki67 (1:100, Thermo Fisher Scientific, RM-9061); mouse anti- $\alpha$-SMA (1:300, MilliporeSigma, A5228); rabbit anti-desmin (1:100, Abcam, Ab15200); band iotinylated lectin from Bandeiraea simplicifolia (L-3759, 1:100, MilliporeSigma). For image quantification, 20 random images at high magnification (40×) of 2 nonconsecutive sections of at least 3 mice per group were quantified using ImageJ software $(\mathrm{NIH})$.

Microarray analyses. For microarray analysis, $6 \times 10^{5} \mathrm{LX} 2$ cells were cultured in DMEM supplemented with $10 \%$ fetal bovine serum in a $10 \mathrm{~cm}$ plate and infected with Adenovirus-hGata4 (abm, 093375A) or Adenovirus-GFP (abm, 000541A) at a MOI of 100 over 72 hours. Gene profile was performed in 3 independent experiments for LX2 cells infected with Adenovirus-hGata4 or Adenovirus-GFP using Affimetrix GeneChip Human Gene 2.0ST Array. Differentially expressed genes were defined as those for which the nominal $P$ value was 0.05 . Gene expression data are available through the Gene Expression Omnibus database (accession number GSE168818).

Quantitative RT-PCR. Total RNA from mouse livers or LX2 cells was isolated using the RNeasy Plus Micro kit (Qiagen). cDNA was synthesized using the QuantiTect Reverse Transcription Kit (Qiagen, 205311). 
Quantitative RT-PCR analysis was performed using a 7500 Real-Time PCR system (Applied Biosyste$\mathrm{ms}$ ). RNA expression of target genes was normalized based on comparison to the housekeeping gene. The $\Delta \Delta \mathrm{Ct}$ method was used to calculate changes in gene expression levels. Quantitative qPCR was performed with at least 5 independent samples in triplicate. qPCR was performed using the following TaqMan probes (Applied Biosystems): ACTA2 Hs00426835_g1, COL1A1 Hs00164004_m1, LAMA1 Hs00300550_m1, EPAS1 Hs0102649_m1, TLR4 Hs00152939_m1, IL-6 Hs00174131_m1, TIMP1 Hs01092512_g1, ßACT Hs03023880_g1, GATA4 Mm00484689_m1, LAMA1 Mm01226102_m1, COL1A1 Mm00801666_g1, EPAS1 Mm01236112_m1, ACTA2 Mm00725412_s1, VEGF Mm00437306_m1, TGF- $\beta$ Mm01178820_m1, and $\beta$ ACT Mm02619580_g1 (Applied Biosystems). The primers for Sybr green qPCR are listed in Supplemental Table 1.

Epas1 silencing by RNA interference. Specific siRNA for human Epas1 (HSS103261) and Stealth siRNA negative control (12935300) were purchased from Invitrogen. For transfection, 30 pmol of the corresponding siRNA were used for transfecting $2 \times 10^{5}$ LX2 cells cultured in 6-well plates using Lipofectamine 2000 (Invitrogen). After 72 hours of incubation, cells were collected for RNA isolation and qPCR analyses.

ChIP. ChIP assays were performed using Dynabeads Protein A (100.01D, Invitrogen) following the manufacturer's recommendations, with some modifications. LX2 cells were grown in DMEM supplemented with $10 \%$ FBS on a $15 \mathrm{~cm}$ plate to approximately $7 \times 10^{6}$ cells and were infected with Ad-GATA4 at a MOI of 100 . After 72 hours, cells were treated with 1\% formaldehyde for 30 minutes to crosslink protein-DNA complexes. Cells were lysed and sonicated to shear the DNA. The cleared supernatant was divided into 2 samples. One of the samples was incubated with $5 \mu \mathrm{g}$ anti-GATA4 antibody (Santa Cruz Biotechnology, SC-25310) and the other sample was incubated with $5 \mu \mathrm{g}$ IgG (Santa Cruz Biotechnology) as a nonspecific antibody for 2 hours at room temperature. Following incubation in $5 \mathrm{M} \mathrm{NaCl}$ at $65^{\circ} \mathrm{C}$ overnight to reverse the crosslinks, DNA was recovered by phenol-chloroform extraction. For Chip-qPCR of EPAS1 the following primers were used: 5'-GTTGCTGGACACACCACATTTC-3' and 5'-CCCTAAGTCCCGGGTACAG-3'. Percentage input method was used to calculate enrichment of GATA4 binding to EPAS1 DNA.

CCl4-induced liver fibrosis and adenovirus treatment. Three- to four-month-old female and male C57Bl/ 6 mice were treated with intraperitoneal injections of $0.5 \mathrm{~mL} / \mathrm{kg}$ of body weight $\mathrm{CCl}_{4}$ (319961-1L, MilliporeSigma) dissolved in olive oil (1:1) (O1514, $500 \mathrm{~mL}$, MilliporeSigma) twice per week for 4 weeks ( 8 injections total) to induce liver fibrosis. For adenovirus treatment, mice were injected via tail vein with $1.8 \times 10^{9}$ infection unit (IFU) of Ad-GATA4 or Ad-GFP as a control 48 hours after the last $\mathrm{CCl}_{4}$ injection. Mice were sacrificed for analysis 1 week after the adenoviral injection.

For Gata4 inactivation in adult liver, Gata4-floxed mice were injected via the tail vein with $4.6 \times 10^{8}$ IFU Ad-Cre (SignaGen Laboratories, SL100707) or Ad-GFP as control. Mice were sacrificed for analyses 2 weeks after adenoviruses treatment.

Cloning, cell culture, transfection experiments, and reporter assays. An $810 \mathrm{pb}$ fragment of the human EPAS1 gene was generated by PCR using the following 2 primers: forward 5'-AATCAGGATGGTACCTAGAAGCATAT-3' and reverse 5'-GCCTAAAACAGCTCGAGGATCAGCACC-3'. This fragment was then cloned into pGL3 reporter vector (Promega) containing the thymidine kinase minimal promoter to generate the pGL3-EPAS1 wt vector. Mutations of the GATA sites in the EPAS1 fragment were introduced by PCR using the following primers: forward 5'-TTACCCAGATCTTAAGCTTAGGAAAG-3' and reverse 5'-CTTTCCTAAGCTTAAGATCTGGGTAA-3'. The EPAS1 fragment containing the mutated GATA sites was cloned into pGL3 reporter vector to generate the pGL3-EPAS1 mut. 293T cells were maintained in DMEM supplemented with 10\% FBS. Cells were cultured in 6-well plates at a density of $2 \times 10^{5}$ cells per well and transfected using Lipofectamine 2000 (Promega) following the manufacturer's recommendations. Each well was transfected with $2.5 \mu \mathrm{g}$ luciferase reporter plasmids. The firefly luciferase activity was normalized to renilla luciferase activity by cotransfecting each well with $0.5 \mu \mathrm{g}$ pRL-renilla reporter vector (Promega, E1910). Cells were collected 72 hours after transfection, and the luciferase activity was measured using the Dual Luciferase Kit Assay (Promega) and detected in a luminometer (Glomax 20/20, Promega).

Western blot. Cells and liver samples were lysed and homogenized in Protease Inhibitor buffer (S8820, MilliporeSigma). Protein concentration was determined using Quick Start Bradford 1X Dye Reagent (5000205, Bio-Rad). Electrophoretically separated proteins were transferred to PVDF membranes (IPFL00010, MilliporeSigma) and incubated with the following primary antibodies: mouse anti- $\alpha$-SMA (1:1000, MilliporeSigma, A5228), rabbit anti-collagen IV (1:1000, Abcam, Ab227616), mouse anti-GATA4 (1:1000, R\&D Systems, MAB2606), rabbit anti-SMAD7 (1:100, Invitrogen, 42-0400), mouse anti-phosphoSTAT1 (Tyr701) (1:500, 
Invitrogen, 33-3400), rabbit anti-STAT1 (1:1000, Cell Signaling, 9172), rabbit anti-GAPDH (1:15,000, Cell Signaling, 2128), and mouse anti- $\beta$-actin (1:10,000, MilliporeSigma, A5441). Secondary antibodies rabbit antiIRDye 800CW (LI-COR, 925-32211), mouse anti-IRDye 680RD (925-68070), mouse anti-IgG-peroxidase (MilliporeSigma A9044), and rabbit anti-IgG peroxidase (MilliporeSigma A0545) were used. The Odyssey CLx Infrared Imaging System (LICOR) or enhanced chemiluminescence detection reagents (GE Healthcare Bio-Sciences Corp.) in the Image Quant 800 System (Amersham) was used for imaging the blots.

Statistics. Statistical analyses were performed using GraphPad Prism (version 7.0) and the 2-tailed Student's test or 2-way ANOVA. $P<0.05$ was considered statistically significant.

Study approval. All experiments using animals complied with institutional guidelines RD 53/2013 and the EU Directive 2010/63/EU and were reviewed and approved by the Institutional Animal Care and Use Committee of the University of Seville (Seville, Spain).

\section{Author contributions}

LV, NA, ID, RC, MRR, and MGT performed the experiments. LP, RMC, FM, DAC, and AR procured and analyzed the data. DAC and AR wrote the manuscript. All authors edited the manuscript. AR is the guarantor of this work and, as such, had full access to all the data in the study and takes responsibility for the integrity of the data and the accuracy of the data analysis.

\section{Acknowledgments}

This work was supported by grants from the Spanish Ministry of Economy and Innovation (MICIN/ AEI/10.13039/501100011033/ FEDER) (BFU2017-82497-P-to AR and BFU2017-83907-P to RMC and RC); from Spanish Ministry of Science and Innovation (MICIN/AEI/10.13039/501100011033) (PID2020114656RB-I00 to AR); from Agencia Andaluza del Conocimiento, Consejería de Transformación Económica, Industria, Conocimiento y Universidades (PY20_00850 to AR); and from ISCIII-Subdirección General de Evaluación y Fomento de la Investigación cofunded with Fondos FEDER (PI16/00175 to DAC). We thank Antonio Cárdenas and Ana Belén Hitos for their technical assistance and Eloisa Andújar and Mónica Pérez from their technical services in the Genomic Unit at CABIMER.

Address correspondence to: Anabel Rojas, Avda Americo Vespucio, 41092 Sevilla, Spain. Phone: 34.954.467.427; Email: anabel.rojas@cabimer.es.

1. Bataller R, Brenner DA. Liver fibrosis. J Clin Invest. 2005;115(2):209-218.

2. Bataller R, Brenner DA. Hepatic stellate cells as a target for the treatment of liver fibrosis. Semin Liver Dis. 2001;21(3):437-451

3. Kisseleva T, Brenner DA. Fibrogenesis of parenchymal organs. Proc Am Thorac Soc. 2008;5(3):338-342.

4. Reeves HL, Friedman SL. Activation of hepatic stellate cells--a key issue in liver fibrosis. Front Biosci. 2002;7:d808-d826

5. Wong $\mathrm{L}$, et al. Induction of beta-platelet-derived growth factor receptor in rat hepatic lipocytes during cellular activation in vivo and in culture. J Clin Invest. 1994;94(4):1563-1569.

6. Kisseleva T, Brenner DA. Role of hepatic stellate cells in fibrogenesis and the reversal of fibrosis. J Gastroenterol Hepatol. 2007;22 Suppl 1:S73-S78.

7. Troeger JS, et al. Deactivation of hepatic stellate cells during liver fibrosis resolution in mice. Gastroenterology. 2012;143(4):1073-1083

8. Kisseleva T, et al. Myofibroblasts revert to an inactive phenotype during regression of liver fibrosis. Proc Natl Acad Sci U S A. 2012;109(24):9448-9453.

9. Liu X, et al. Identification of lineage-specific transcription factors that prevent activation of hepatic stellate cells and promote fibrosis resolution. Gastroenterology. 2020;158(6):1728-1744.

10. Delgado I, et al. GATA4 loss in the septum transversum mesenchyme promotes liver fibrosis in mice. Hepatology. 2014;59(6):2358-2370

11. Shayakhmetov DM, et al. Analysis of adenovirus sequestration in the liver, transduction of hepatic cells, and innate toxicity after injection of fiber-modified vectors. $J$ Virol. 2004;78(10):5368-5381.

12. Bosse T, et al. Gata4 is essential for the maintenance of jejunal-ileal identities in the adult mouse small intestine. Mol Cell Biol. 2006;26(23):9060-9070

13. Zhang Y, et al. Protective role of estrogen-induced miRNA-29 expression in carbon tetrachloride-induced mouse liver injury. J Biol Chem. 2012;287(18):14851-14862.

14. Xu JW, et al. Estrogen reduces CCL4- induced liver fibrosis in rats. World J Gastroenterol. 2002;8(5):883-887.

15. Rojas A, et al. GATA4 is a direct transcriptional activator of cyclin D2 and Cdk4 and is required for cardiomyocyte proliferation in anterior heart field-derived myocardium. Mol Cell Biol. 2008;28(17):5420-5431.

16. Kim WY, et al. Failure to prolyl hydroxylate hypoxia-inducible factor alpha phenocopies VHL inactivation in vivo. EMBO J. 2006;25(19):4650-4662.

17. Nakano Y, et al. A deactivation factor of fibrogenic hepatic stellate cells induces regression of liver fibrosis in mice. Hepatology. 
2020;71(4):1437-1452.

18. Zheng R, et al. Function of GATA factors in the adult mouse liver. PLoS One. 2013;8(12):e83723.

19. Geraud C, et al. GATA4-dependent organ-specific endothelial differentiation controls liver development and embryonic hematopoiesis. J Clin Invest. 2017;127(3):1099-1114.

20. Mohlin S, et al. PI3K-mTORC2 but not PI3K-mTORC1 regulates transcription of HIF2A/EPAS1 and vascularization in neuroblastoma. Cancer Res. 2015;75(21):4617-4628.

21. Dai YS, et al. The transcription factors GATA4 and dHAND physically interact to synergistically activate cardiac gene expression through a p300-dependent mechanism. J Biol Chem. 2002;277(27):24390-24398

22. Svensson EC, et al. A functionally conserved N-terminal domain of the friend of GATA-2 (FOG-2) protein represses GATA4-dependent transcription. J Biol Chem. 2000;275(27):20762-20769.

23. Qu A, et al. Hypoxia-inducible transcription factor $2 \alpha$ promotes steatohepatitis through augmenting lipid accumulation, inflammation, and fibrosis. Hepatology. 2011;54(2):472-483.

24. Cai $\mathrm{H}$, et al. Hypoxia-inducible factor-2 promotes liver fibrosis in non-alcoholic steatohepatitis liver disease via the NF- $\kappa \mathrm{B}$ signalling pathway. Biochem Biophys Res Commun. 2021;540:67-74.

25. Copple BL, et al. Hypoxia-inducible factor-1 $\alpha$ regulates the expression of genes in hypoxic hepatic stellate cells important for collagen deposition and angiogenesis. Liver Int. 2011;31(2):230-244.

26. Xiong A, Liu Y. Targeting hypoxia inducible factors- $1 \alpha$ as a novel therapy in fibrosis. Front Pharmacol. 2017;8:326

27. Yang L, et al. Vascular endothelial growth factor promotes fibrosis resolution and repair in mice. Gastroenterology. 2014;146(5):1339-1350

28. Menendez-Montes I, et al. Myocardial VHL-HIF signaling controls an embryonic metabolic switch essential for cardiac maturation. Dev Cell. 2016;39(6):724-739.

29. Pu WT, et al. GATA4 is a dosage-sensitive regulator of cardiac morphogenesis. Dev Biol. 2004;275(1):235-244.

30. Srinivas S, et al. Cre reporter strains produced by targeted insertion of EYFP and ECFP into the ROSA26 locus. BMC Dev Biol. 2001;1:4. 